\title{
Determinants of bioelectrical phase angle in disease
}

\author{
Nicole Stobäus ${ }^{1}$, Matthias Pirlich ${ }^{2}$, Luzia Valentini ${ }^{3}$, Jörg Dieter Schulzke ${ }^{1}$ and Kristina Norman ${ }^{3 *}$ \\ ${ }^{1}$ Department of General Medicine, Charité-Universitätsmedizin Berlin, Campus Benjamin Franklin, Germany \\ ${ }^{2}$ Department of Internal Medicine, Evangelische Elisabeth Klinik, Berlin, Germany \\ ${ }^{3}$ Department of Gastroenterology, Hepatology and Endocrinology, Charité-Universitätsmedizin Berlin, Charité Campus Mitte \\ (CCM), Charitéplatz 1, 10117 Berlin, Germany
}

(Submitted 15 April 2011 - Final revision received 22 June 2011 - Accepted 24 June 2011 - First published online 28 September 2011)

\section{Abstract}

Phase angle (PhA), a parameter of bioelectrical impedance analysis, is a well-known predictor of morbidity and mortality in various diseases. The causes of decreased PhA are, however, not yet completely understood. We therefore investigated determinants of PhA in 777 hospitalised patients in a retrospective analysis. PhA was assessed by bioelectrical impedance analysis at $50 \mathrm{KHz}$. Subjective global assessment (SGA) was used to evaluate nutritional status. Age, sex, BMI as well as nutritional status (SGA), benign or malignant disease and C-reactive protein (CRP) were investigated as potential determinants of PhA and standardised PhA (SPhA) $=(\mathrm{Observed} \mathrm{PhA}-$ mean PhA of reference values)/standard deviation of reference values in a general linear model regression analysis. Next to age (estimated effect size, $46.6 \%$; $P<0.0001)$, malnutrition $(39.1 \% ; P<0.0001)$ emerged as a major PhA determinant in our study population. Moreover, sex (6.4\%; $P<0.0001)$, CRP $(4.4 \% ; P<0.0001)$ and BMI $(3.5 \% ; P<0.0001)$ exhibited a significant influence on PhA, whereas malignant disease showed no significant effect in this model. The only significant determinants of SPhA were malnutrition $(85 \cdot 4 \% ; P<0 \cdot 0001)$ and inflammation $(9.6 \% ; P<0.0001)$. In conclusion, next to the established predictors, malnutrition and inflammation have a strong impact on PhA in sick individuals, which partly explains its prognostic power. When investigating the SPhA, only malnutrition and inflammation were found to be significant predictors, as a result of which the SPhA is considered a more suitable indicator of nutritional and health status.

\section{Key words: Bioelectrical impedance analysis: Phase angle: Standardised phase angle: Malnutrition: Inflammation}

Bioelectrical impedance analysis is a portable, easyto-perform, non-invasive bedside method, which allows an assessment of body composition in both ambulatory and hospitalised patients. Since the calculation of body composition is limited in several clinical conditions, the consideration of raw bioelectrical impedance analysis parameters has gained increasing attention. Moreover, phase angle (PhA) is an excellent predictor of morbidity and mortality in HIV/AIDS, pancreatic, colorectal, breast and lung cancer, liver cirrhosis, dialysis, pulmonary disease, amyotrophic lateral sclerosis, geriatric and surgical patients ${ }^{(1-16)}$. PhA is calculated from resistance ( $R$, pure opposition of a biological conductor to alternating electric current) and reactance $\left(X_{\mathrm{c}}\right.$, capacitive resistance of cell membranes).

PhA primarily reflects the electrical integrity of vital cell membranes $^{(1)}$ and indicates the distribution of water between intracellular and extracellular space ${ }^{(2)}$. Theoretically, changes in reactance associated with variability in cell size, membrane permeability or intracellular composition may contribute to the variation among individuals in $\mathrm{PhA}$ at a fixed frequency ${ }^{(17)}$. Higher PhA suggests large quantities of intact cell membranes ${ }^{(9)}$, while lower PhA suggests cell death or decreased cell integrity. PhA is decreased in patients with significant weight loss ${ }^{(8,18-20)}$ and is considered a marker of clinically relevant malnutrition characterised by both increased extracellular fluid and decreased body cell mass ${ }^{(9)}$.

In healthy subjects, age, sex and BMI are the major determinants of $\mathrm{PhA}^{(21-24)}$. Ageing is associated with decline in tissue mass, which results in decreasing PhA. Males have higher PhA values due to higher muscle mass. Higher BMI is associated with more cells, which increases PhA until a BMI of $30 \mathrm{~kg} / \mathrm{m}^{2}$. Interestingly, at BMI $>40 \mathrm{~kg} / \mathrm{m}^{2}$, an inverse relationship with PhA is observed. This has been attributed to increased tissue hydration or a pathological fluid overload ${ }^{(24)}$.

PhA is useful in clinical practice, since it allows identification of patients at risk of impaired nutritional status

Abbreviations: CRP, C-reactive protein; PhA, phase angle; $R$, resistance; SGA, subjective global assessment; SPhA, standardised phase angle; $X_{\mathrm{c}}$, capacitive reactance.

*Corresponding author: Dr K. Norman, fax +49 30450514 923, email kristina.norman@charite.de 
and decreased survival. However, what contributes to a low $\mathrm{PhA}$ in disease is not yet completely understood. We therefore investigated the determinants of $\mathrm{PhA}$ in a retrospective sample of hospitalised patients and, moreover, studied the risk factors for patients' individual deviation from age-, sex- and BMIstratified reference values (standardised PhA, SPhA) ${ }^{(24)}$.

\section{Patients and methods}

We pooled data from 777 patients with full data sets from prospective cross-sectional studies ${ }^{(8,25-27)}$ and previously unpublished results with the same method (N Stobäus, unpublished results). Patients admitted to the Charité University Hospital, Berlin, Germany aged $>18$ years were originally consecutively recruited. The study protocols were approved by the Ethics Committee of the Charité, and all studies were conducted according to the guidelines laid down in the Declaration of Helsinki. All subjects signed informed consent forms. Age, sex, weight and height as well as clinical variables were recorded.

\section{Bioelectrical impedance analysis}

Bioelectrical impedance analysis was performed at $50 \mathrm{kHz}$ and $0.8 \mathrm{~mA}$ by experienced observers according to a standardised protocol described elsewhere ${ }^{(28)}$, using a Nutrigard M (Data Input, Darmstadt, Germany). $R$ and $X_{\mathrm{c}}(\Omega)$ were measured and $\mathrm{PhA}$ was calculated $\left(\operatorname{PhA}\left(^{\circ}\right)=\left(X_{\mathrm{C}} / R\right) \times(180 / \pi)\right)$. The percentage of patients with PhA below the fifth percentile of age-, sex- and BMI-stratified reference values was determined $^{(24)}$. PhA was also standardised according to the reference values: $\mathrm{SPhA}=($ observed $\mathrm{PhA}-$ mean $\mathrm{PhA}) / \mathrm{SD}$, where $\mathrm{SD}$ is standard deviation of PhA, where mean and SD are from age-, sex- and BMI-stratified reference values.

\section{Assessment of nutritional status}

Weight and height were measured to calculate BMI: weight $(\mathrm{kg}) /$ height $\left(\mathrm{m}^{2}\right)$. Nutritional status was assessed by subjective global assessment $(\mathrm{SGA})^{(29)}$. Patients were classified as well nourished (SGA A), moderately malnourished (SGA B) or severely malnourished (SGA C).

\section{Inflammation}

C-reactive protein (CRP) was determined according to standard methods (mg/l).

\section{Statistical analysis}

Statistical analysis was performed with PASW Statistics 18 (SPSS, Inc., Chicago, IL, USA). Data are presented as means and standard deviations. Comparison between groups was made using Student's $t$ test and $\chi^{2}$ test. The relationship between PhA and its determinants was described by Pearson's correlation. Regression analysis was performed with the general linear model allowing adjustment for continuous and categorical variables, in order to assess the impact of age, sex, BMI, nutritional status defined by SGA, disease (malignant/ benign) and inflammation on PhA. The estimates of the effect size are expressed as percentages of $\eta^{2}$. A $P$ value $<0.05$ was considered significant.

\section{Results}

A total of 777 patients ( 367 men) were included in the pooled analysis. Mean age was 53.6 (SD 16.7) years and women were significantly older $(P=0.006)$. According to the BMI, $10 \%$ were underweight while $37.8 \%$ were overweight or obese; $54.8 \%$ were considered moderately or severely malnourished by SGA. Patients with tumours (34\%) were older (63.3 (SD 12.6) v. 48.5 (SD 16.3) years, $P<0.001$ ) and had a higher BMI than patients with benign disease (24.7 (SD 4.7) $v .23 .8$ (SD 4.9$) \mathrm{kg} / \mathrm{m}^{2}, P=0.01$ ). Mean length of hospital stay was $13 \cdot 1$ (sD 11.5) d.

Mean PhA was $4.91 \pm 1.17^{\circ}$, ranging from 1.62 to $8.51^{\circ}$, and was significantly higher in male patients; $41 \%$ of whom exhibited PhA beneath the fifth percentile of age-, sex- and BMIstratified reference values. SPhA ranged from -7.2 to $2.5 \mathrm{SD}$, $56.5 \%$ of patients had PhA below $-1 \mathrm{SD}$ of the population average. PhA correlated inversely with age (men: $r-0.479$, $P<0.001$; women: $r-0.503, P<0.001$ ), while only a weak positive correlation existed between PhA and BMI, which was significant in women $(r 0 \cdot 116, P=0 \cdot 019)$.

PhA and SPhA were significantly decreased in moderate and severe malnutrition. Patients with benign disease had higher

Table 1. Results from the general linear model regression analysis determinants of phase angle and standardised phase angle ( $\beta$ Coefficients and percentages)

\begin{tabular}{|c|c|c|c|c|c|c|}
\hline & \multicolumn{3}{|c|}{ Phase angle } & \multicolumn{3}{|c|}{ Standardised phase angle } \\
\hline & $\beta$ & $\begin{array}{l}\text { Estimates of } \\
\text { effect size (\%) }\end{array}$ & $P$ & $\beta$ & $\begin{array}{l}\text { Estimates of } \\
\text { effect size (\%) }\end{array}$ & $P$ \\
\hline Age (years) & -0.033 & $46 \cdot 6$ & $<0.0001$ & -0.006 & $2 \cdot 3$ & NS \\
\hline Moderate malnutrition & -0.538 & $12 \cdot 6$ & $<0.0001$ & -0.743 & $27 \cdot 2$ & $<0.0001$ \\
\hline Severe malnutrition & -0.935 & $26 \cdot 5$ & $<0.0001$ & -1.307 & $58 \cdot 2$ & $<0.0001$ \\
\hline Male sex & 0.314 & $6 \cdot 4$ & $<0.0001$ & -0.165 & $2 \cdot 1$ & NS \\
\hline CRP (mg/l) & -0.003 & 4.4 & $<0.0001$ & -0.004 & $9 \cdot 6$ & $<0.0001$ \\
\hline BMI $\left(\mathrm{kg} / \mathrm{m}^{2}\right)$ & 0.026 & 3.5 & $<0.0001$ & 0.008 & 0.4 & NS \\
\hline Malignant disease & 0.043 & 0.0 & NS & 0.078 & 0.3 & NS \\
\hline
\end{tabular}

CRP, C-reactive protein. 
PhA than patients with tumours $\left(5.14 \pm 1.14^{\circ} v .4 .48 \pm 1.09^{\circ}\right.$, $P<0 \cdot 001)$. CRP correlated inversely with PhA $(r-0 \cdot 248$, $P<0 \cdot 001)$.

\section{Determinants}

Age, sex, BMI, nutritional status (SGA), inflammation and malignant $v$. benign disease were investigated as possible determinants of PhA and SPhA in a general linear model regression analysis (Table 1 ). Next to age, malnutrition emerged as a major determinant of PhA. Moreover, sex, CRP and BMI significantly influenced PhA, whereas diagnosis showed no effect in this model. When investigating SPhA, the only significant determinants were malnutrition and CRP.

Interestingly, after stratifying the patients according to CRP (no inflammation, $<5 \mathrm{mg} / \mathrm{l}$; low to moderate, $5-50 \mathrm{mg} / \mathrm{l}$; high, $>50 \mathrm{mg} / \mathrm{l}$ ), the association between CRP and SPhA was statistically significant only in patients with low-to-moderate inflammation, whereas SGA exerted a significant impact in all CRP categories (data not shown).

\section{Discussion}

We pooled data from cross-sectional studies in order to investigate the determinants of PhA in disease. Age, malnutrition, sex, inflammation and BMI were identified as significant predictors of PhA. When PhA was standardised according to age-, sex- and BMI-stratified reference values, only moderate and severe malnutrition, as well as inflammation, were significant risk factors.

Age, sex ${ }^{(21,30)}$ and $\mathrm{BMI}^{(22)}$ are the strongest predictors in healthy populations. Few studies have, however, investigated the risk factors for decreased PhA in disease. In haemodialysis, creatinine and log-soluble leptin receptor:leptin ratio ${ }^{(31)}$ as well as albumin, mid-arm muscle circumference, SGA and normalised protein catabolic rate ${ }^{(32)}$ were significant independent predictors of PhA. Moreover, IL-6 predicted lower PhA as well as greater loss of PhA over time in haemodialysis patients ${ }^{\text {(33) }}$.

In the present analysis, age exerted the largest impact on PhA, explaining $46.6 \%$ of its variability. Ageing is associated with loss of muscle mass (decline in $X_{\mathrm{c}}$ ) and decrease in total body water (increase in $R$ ), which results in decreased PhA. This inverse relationship was also found in healthy ${ }^{(21-24)}$ and ill populations ${ }^{(34)}$. PhA was reduced in malnutrition, which is in accordance with previous findings ${ }^{(8,18,19)}$. The impact of CRP is expected, as several studies have shown an association between PhA and inflammation ${ }^{(24,33,35-37)}$

However, only malnutrition and inflammation predicted SPhA. Transforming PhA into a $Z$-score allows assessment of individual deviations from age-, sex- and BMI-specific population average, which clearly enhances its validity. SPhA has been shown to be the strongest predictor of 6-month mortality in tumour patients ${ }^{(8)}$, and of increased post-operative complications ${ }^{(16)}$.

The present results are limited since they are a retrospective analysis of prospectively collected data. This precluded the assessment of potentially interesting parameters such as physical activity, probably a contributing factor as suggested by earlier findings in healthy individuals ${ }^{(22,38)}$. However, to what extent a disease-specific impact might override the influence of physical activity in patients is not known. Future studies should include the assessment of physical activity when studying PhA in disease.

In conclusion, together with the known biological determinants, malnutrition and inflammation had a strong impact on PhA in disease. However, only malnutrition and inflammation predicted SPhA. As a result, SPhA, the individual deviation from population average, is therefore considered a more suitable indicator of nutritional and inflammatory status.

\section{Acknowledgements}

K. N. and N. S. designed the study and performed the analysis. L. V., M. P. and J. D. S. contributed the data and significant advice. N. S. and K. N. wrote the manuscript. The authors have no conflicts of interest. The present study received no specific grant from any funding agency in the public, commercial or not-for-profit sectors.

\section{References}

1. Ott M, Fischer H, Polat H, et al. (1995) Bioelectrical impedance analysis as a predictor of survival in patients with human immunodeficiency virus infection. I Acquir Immune Defic Syndr Hum Retrovirol 9, 20-25.

2. Schwenk A, Beisenherz A, Romer K, et al. (2000) Phase angle from bioelectrical impedance analysis remains an independent predictive marker in HIV-infected patients in the era of highly active antiretroviral treatment. Am J Clin Nutr 72, 496-501.

3. Toso S, Piccoli A, Gusella M, et al. (2000) Altered tissue electric properties in lung cancer patients as detected by bioelectric impedance vector analysis. Nutrition 16, 120-124.

4. Gupta D, Lis CG, Dahlk SL, et al. (2004) Bioelectrical impedance phase angle as a prognostic indicator in advanced pancreatic cancer. Br J Nutr 92, 957-962.

5. Gupta D, Lammersfeld CA, Burrows JL, et al. (2004) Bioelectrical impedance phase angle in clinical practice: implications for prognosis in advanced colorectal cancer. Am J Clin Nutr 80, 1634-1638.

6. Gupta D, Lammersfeld CA, Vashi PG, et al. (2008) Bioelectrical impedance phase angle as a prognostic indicator in breast cancer. BMC Cancer $\mathbf{8}, 249$.

7. Gupta D, Lammersfeld CA, Vashi PG, et al. (2009) Bioelectrical impedance phase angle in clinical practice: implications for prognosis in stage IIIB and IV non-small cell lung cancer. BMC Cancer 9, 37.

8. Norman K, Stobäus N, Zocher D, et al. (2010) Cutoff percentiles of bioelectrical phase angle predict functionality, quality of life, and mortality in patients with cancer. Am J Clin Nutr 92, 612-619.

9. Selberg O \& Selberg D (2002) Norms and correlates of bioimpedance phase angle in healthy human subjects, hospitalized patients, and patients with liver cirrhosis. Eur J Appl Physiol 86, 509-516.

10. Chertow GM (1997) Phase angle predicts survival in hemodialysis patients. J Renal Nutr 7, 204-207.

11. Fein PA, Gundumalla G, Jorden A, et al. (2002) Usefulness of bioelectrical impedance analysis in monitoring nutrition status and survival of peritoneal dialysis patients. Adv Perit Dial 18, 195-199. 
12. Mushnick R, Fein PA, Mittman N, et al. (2003) Relationship of bioelectrical impedance parameters to nutrition and survival in peritoneal dialysis patients. Kidney Int Suppl S53-S56.

13. Krause L, Becker MO, Brueckner CS, et al. (2010) Nutritional status as marker for disease activity and severity predicting mortality in patients with systemic sclerosis. Ann Rheum Dis 69, 1951-1957.

14. Desport JC, Marin B, Funalot B, et al. (2008) Phase angle is a prognostic factor for survival in amyotrophic lateral sclerosis. Amyotroph Lateral Scler 9, 273-278.

15. Wirth R, Volkert D, Rosler A, et al. (2010) Bioelectric impedance phase angle is associated with hospital mortality of geriatric patients. Arch Gerontol Geriatr 51, 290-294.

16. Barbosa-Silva MC \& Barros AJ (2005) Bioelectric impedance and individual characteristics as prognostic factors for postoperative complications. Clin Nutr 24, 830-838.

17. Baumgartner RN, Chumlea WC \& Roche AF (1988) Bioelectric impedance phase angle and body composition. Am J Clin Nutr 48, 16-23.

18. Gupta D, Lis CG, Dahlk SL, et al. (2008) The relationship between bioelectrical impedance phase angle and subjective global assessment in advanced colorectal cancer. Nutr J 7, 19 .

19. Norman K, Smoliner C, Valentini L, et al. (2007) Is bioelectrical impedance vector analysis of value in the elderly with malnutrition and impaired functionality? Nutrition $\mathbf{2 3}$, $564-569$.

20. Oliveira CM, Kubrusly M, Mota RS, et al. (2010) The phase angle and mass body cell as markers of nutritional status in hemodialysis patients. J Ren Nutr 20, 314-320.

21. Barbosa-Silva MC, Barros AJ, Wang J, et al. (2005) Bioelectrical impedance analysis: population reference values for phase angle by age and sex. Am J Clin Nutr 82, 49-52.

22. Dittmar M (2003) Reliability and variability of bioimpedance measures in normal adults: effects of age, gender, and body mass. Am J Phys Anthropol 122, 361-370.

23. Kyle UG, Genton L, Slosman DO, et al. (2001) Fat-free and fat mass percentiles in 5225 healthy subjects aged 15 to 98 years. Nutrition 17, 534-541.

24. Bosy-Westphal A, Danielzik S, Dorhofer RP, et al. (2006) Phase angle from bioelectrical impedance analysis: population reference values by age, sex, and body mass index. JPEN J Parenter Enteral Nutr 30, 309-316.

25. Norman K, Schutz T, Kemps M, et al. (2005) The Subjective Global Assessment reliably identifies malnutrition-related muscle dysfunction. Clin Nutr 24, 143-150.
26. Norman K, Smoliner C, Kilbert A, et al. (2008) Diseaserelated malnutrition but not underweight by BMI is reflected by disturbed electric tissue properties in the bioelectrical impedance vector analysis. Br J Nutr 100, 590-595.

27. Valentini L, Wirth EK, Schweizer U, et al. (2009) Circulating adipokines and the protective effects of hyperinsulinemia in inflammatory bowel disease. Nutrition 25, 172-181.

28. Kyle UG, Bosaeus I, De Lorenzo AD, et al. (2004) Bioelectrical impedance analysis-part II: utilization in clinical practice. Clin Nutr 23, 1430-1453.

29. Detsky AS, McLaughlin JR, Baker JP, et al. (1987) What is subjective global assessment of nutritional status? JPEN J Parenter Enteral Nutr 11, 8-13.

30. Piccoli A, Pillon L \& Dumler F (2002) Impedance vector distribution by sex, race, body mass index, and age in the United States: standard reference intervals as bivariate $Z$ scores. Nutrition 18, 153-167.

31. Beberashvili I, Sinuani I, Azar A, et al. (2009) Nutritional and inflammatory status of hemodialysis patients in relation to their body mass index. $J$ Ren Nutr 19, 238-247.

32. Maggiore Q, Nigrelli S, Ciccarelli C, et al. (1996) Nutritional and prognostic correlates of bioimpedance indexes in hemodialysis patients. Kidney Int 50, 2103-2108.

33. Johansen KL, Kaysen GA, Young BS, et al. (2003) Longitudinal study of nutritional status, body composition, and physical function in hemodialysis patients. Am J Clin Nutr 77, 842-846.

34. Chertow GM, Lazarus JM, Lew NL, et al. (1997) Bioimpedance norms for the hemodialysis population. Kidney Int 52, 1617-1621.

35. Avram MM, Fein PA, Rafiq MA, et al. (2006) Malnutrition and inflammation as predictors of mortality in peritoneal dialysis patients. Int Soc Nephrol 70, 4-7.

36. Gunn SM, Halbert JA, Giles LC, et al. (2008) Bioelectrical phase angle values in a clinical sample of ambulatory rehabilitation patients. Dyn Med 7, 14 .

37. Demirci MS, Demirci C, Ozdogan O, et al. (2011) Relations between malnutrition-inflammation-atherosclerosis and volume status. The usefulness of bioimpedance analysis in peritoneal dialysis patients. Nephrol Dial Transplant 26, $1708-1716$.

38. Marra M, Caldara A, Montagnese C, et al. (2009) Bioelectrical impedance phase angle in constitutionally lean females, ballet dancers and patients with anorexia nervosa. Eur $J$ Clin Nutr 63, 905-908. 